\title{
Glycemic Modulation Resulting from Different Orders of Physical Training in Type 2 Diabetics: A Case Study
}

\author{
Valesca Nayara Silva e Silva, William Valadares Campos Pereira*, Riara Bezerra Pontes de \\ Medeiros, Nathália de Souza Melo Abath Barbosa, Jonathan Nícolas dos Santos Ribeiro, Iago \\ Vilela Dantas and Denise Maria Martins Vancea \\ Department of Diabetes, Sports Exercise of the Brazilian Diabetes Society, Brazil
}

*Corresponding author: William Valadares Campos Pereira, Department of Diabetes, Sports Exercise of the Brazilian Diabetes Society, Brazil.

To Cite This Article: Valesca Nayara Silva e Silva, William Valadares Campos Pereira, Glycemic Modulation Resulting from Different Orders of Physical Training in Type 2 Diabetics: A Case Study. Am J Biomed Sci \& Res. 2021 - 12(2). AJBSR.MS.ID.001732.

DOI: 10.34297/AJBSR.2021.12.00173.

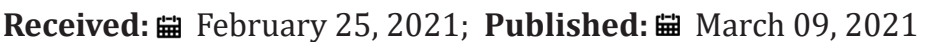

\begin{abstract}
Aerobic training and strength training are recommended for the treatment of diabetes, but it is not clear the effect of the sequence of these trainings in the same session for glycemic modulation of type 2 diabetics. This study aimed to verify the glycemic modulation of type 2 diabetics submitted to different orders of training. It was characterized as a case study, approved by the Ethics and Research Committee 007/09. Five women with type 2 diabetes mellitus (DM2) participated in this study, attending a program of supervised physical exercise for diabetics of a public university in the Northeast/Brazil. They conducted 12 sessions of Combined Training (aerobic and strength in the same session). The program was divided in two moments: first moment: six sessions, where the order of exercises was Aerobic and Strength (AF); second moment: six sessions, where it was Strength and Aerobic (FA). The capillary blood glucose was collected before, during and immediately after each session. The non-parametric ANOVA test was performed for repeated measures and the significance level of $p \leq 0,05$ was adopted. The average of glycemic values was measured with the six days of collection of each training. The AF training presented significant reduction of capillary blood glucose values, when compared to the pre-intervention and post-intervention time $(169 \mathrm{mg} / \mathrm{dL}$ vs $117.3 \mathrm{mg} / \mathrm{dL} ; \Delta=-52.6 \mathrm{mg} / \mathrm{dL} ; \mathrm{p}<0.001)$, in the $\mathrm{AF}$ session there was also significant reduction of these values $(166.6 \mathrm{mg} / \mathrm{dL}$ vs $118.4 \mathrm{mg} / \mathrm{dL} ; \Delta=-48.2 \mathrm{mg} / \mathrm{dL} ; \mathrm{p}<0.001)$. In the intragroup comparison, AF and FA did not show significant difference ( $\mathrm{p}=0.68)$. It is concluded that $\mathrm{AF}$ and FA training are safe and efficient strategies, however, the intragroup analysis showed no difference in glycemic modulation in any of the orders.
\end{abstract}

Keywords: Diabetes; Blood Glucose; Exercise

Abbreviations: DM2: type 2 Diabetes Mellitus; PE: Physical Exercise; PE: Physical Exercise; HbA1C: Glycated Hemoglobin; PROCAPE: Emergency Department of Pernambuco

\section{Introduction}

Type 2 diabetes mellitus (DM2) is a metabolic disease characterized by insulin resistance and deficiencies in insulin secretion, representing $90 \%$ to $95 \%$ of all cases of diabetes mellitus (DM) [1]. The DM2 treatment is aimed at maintaining glycemic rates as close as possible to the established standards of normality, through the association of oral and/or insulin medications, adequacy of diet and regular physical exercises [2]. Specifically, the

practice of physical exercise (PE) is determinant in the prevention of DM2 and in the treatment of all forms of DM. When performed regularly, it has proven to be effective not only in improving blood glucose, reducing insulin resistance and promoting insulin secretion, but also in reducing the risk of cardiovascular disease and obesity in patients with DM2 [3]. Sedentary behavior and overweight have a great impact on glycemic levels, making it 
necessary for type 2 diabetics to be encouraged to participate in PE programs on a regular basis, increasing energy expenditure and consequently, obtaining better glucose control [4]. Diabetics who present a better glycemic control, due to adherence to PE present a decline in the prevalence of chronic complications from diabetes, such as nephropathy, retinopathy, peripheral neuropathy, peripheral vascular disease and diabetic foot $[5,6]$.

As an important part of the treatment, PE can be performed through different training methods, one of them is strength training that presents improvements in strength, bone mineral density, blood pressure, lipid profiles, cardiovascular health, insulin sensitivity and muscle mass [7]. The aerobic training, on the other hand, has effectiveness for metabolic control, anthropometry, glycemic control and blood pressure [8,9]. The primary results in studies that evaluated the effects of strength training in DM2, in important institutions in the treatment of diabetes such as the American Diabetes Association [10] and the Brazilian Society of Diabetes 1, besides other studies, suggest the inclusion of strength and aerobic exercise in the same session. The participation of this population in oriented and supervised EF programs offers remarkable benefits to metabolic control [11], from glucose control to social interaction and knowledge about the disease itself, which helps control [12]. According to Yardley et al. [13], the training session should start with strength exercise, because aerobic training can generate a sharper decrease in glucose during exercise, requiring greater need for carbohydrate supplementation, however, this study was conducted in individuals with type 1 diabetes.

No previous studies were found that examined the acute effects related to the order of training types in the same EF session, and in individuals with DM2. Thus, this study aimed to determine whether the order of training in combined training, strength and aerobic, modulate differently the glycemic response in type 2 diabetics.

\section{Materials and Methods}

This study is characterized as a case study, is part of a larger project "Effects of Aerobic Training, Resisted and Combined on Glycemic Control and Body Composition of Type 2 Diabetics", approved by the Ethics and Research Committee of the University of Pernambuco (CEP/UPE:007/09). It is associated to the Research Group Physical Exercise and Non-Transmissible Chronic Diseases registered in the Research Directory of CNPq.

\section{Sample}

Five randomly selected women diagnosed with type 2 diabetes mellitus (DM2) participated in this study. All were part of Doce Vida - Supervised Physical Exercise Program for Diabetics/ESEF/UPE/ Brazil. The average age of the participants was $69.4 \pm 4.5$ years. All had the aptitude to perform all the strength training protocols $\mathrm{A}$ and $\mathrm{B}$ and all the aerobic training protocol.

\section{Inclusion Criteria}

a) Diagnosis of DM2.

b) To have at least three months of participation in the Sweet Life Program.

c) Perform all protocols A and B of strength training.

\section{Physical Exercise Program}

The participants of the sample performed the combined training (aerobic and strength in the same session). The start time of the activities was from $7 \mathrm{am}$ to $9 \mathrm{am}$, after the routine meal and on alternate days, totaling 12 training sessions for this study. The Program took place in the premises of the School of Physical Education/UPE/Brazil, the Sports Gymnasium and the Biodynamics Laboratory, under the supervision of Physical Education Teachers and academics. The program was divided in two moments:

a) The first moment was composed of six sessions, where the order of the exercises was: Aerobic and Strength.

b) The 2nd moment was composed of six sessions, where the order of the exercises was: Strength and Aerobic.

\section{Strength Training Protocol}

It consisted of resistance exercises performed with free weights (dumbbells and washers) and New Fit Equipment $₫$ machinery. The load progression used the series system until the momentary concentric failure, within the range of 08 to 16 repetitions and with an interval of 45 seconds [14]. Prioritized muscle groups and strength exercises performed.

Protocol A: Prioritized muscle groups: pectoralis major muscle, femoral quadriceps muscle, biceps brachii muscle and deltoid muscle. Exercises performed: flying, extension chair, alternate thread with dumbbells and high paddling with dumbbells.

Protocol B: Large dorsal muscle, triceps sural muscle, biceps femoris and triceps brachii muscle.

Exercises performed: low paddling, bilateral plantar flexion, knee flexion with shin guard and triceps pulley.

\section{Aerobic Training Protocol}

There was a 20 -minute walk on a treadmill. To determine the intensity and knowledge of the cardiovascular condition, we used the values of the maximum effort test previously performed in the ergometry sector of the Emergency Department of Pernambuco (PROCAPE) that used Bruce's protocol in the technique. We use a target training area of 50 to $75 \%$ of the reserve heart rate (FCH $=$ [maximum HR - resting HR] x intensity + resting HR) [15]. The heartbeats were obtained and checked by palpation of the radial artery, positioning the index and middle fingers in the distal radius, 
in the left wrist, counted by the evaluator in ten seconds and multiplied by six [16].

\section{Combined Training Protocol}

It consisted of 20 minutes of aerobic training (trekking) in moderate intensity and 20 minutes of strength training, this was divided into protocols $\mathrm{A}$ and $\mathrm{B}$ performing alternation between the training sessions. The combined training protocol took place as follows:

a) Warm up: stretching exercises (15 minutes).

b) Main part: walking (20 minutes) and strength training (protocol A) and in the following session, strength training (protocol B), followed by 20 minutes of walking.

c) Cooling: stretching activities, relaxation and body consciousness work (10 minutes).

\section{Measurement of Capillary Blood Glucose}

The measurement of capillary blood glucose was performed to identify its values and ensure the safety of the volunteers, preventing them from performing the protocols in the presence of hypoglycemia or hyperglycemia before them, during (after the 20 minutes of the first training) and immediately after each combined training session. For this measure, a glucometer and reagent strips of the Contour TS model, of the BAYER brand, respectively, were used. The collection was always performed on the minimum or ring fingers, discarding the first drop of blood and using the second drop [17]. The infectious material (gloves, lancets, tapes and paper towels) used for collection was deposited in a specific box of hospital material. All diabetics who participated in the program should be within the recommended blood glucose level $<200 \mathrm{mg} /$ $\mathrm{dL}$ at the beginning of each session and $>100 \mathrm{mg} / \mathrm{dL}$ at the end of each session [1].

\section{Statistical Analysis}

The non-parametric ANOVA test was performed for repeated measurements. The program used was SPSS 17.0. For this sample, a significance level of $\mathrm{p} \leq 0.05$ was used. The average of 6 training sessions for each order was performed. And to calculate the delta variation the difference between moment 1 and moment 2 was considered.

\section{Results}

It was observed that the sessions of exercises in the order of training AF, presented significant reduction of the glycemic values in the moments: PRE/DURING and PRE/POST training, it was adopted significance level $p<0.001$; (Table 1 ). When the volunteers performed the training sessions in the FA order, there was also a significant reduction in all moments: PRE/DURING; DURING/ POST and PRE/POST ( $\mathrm{p}<0.001$ ); (Table 1). However, no significant difference in glycemic values was observed when the intragroup analysis was performed: $\mathrm{AF}$ and $\mathrm{FA}(\mathrm{p}=0.68)$.

Table 1: Averages of glycemia's pre, During and post training session AF and FA.

\begin{tabular}{|c|c|c|c|c|c|c|}
\hline & Glycemia pre/During & $\mathbf{p} / \boldsymbol{\Delta}$ & Glycemia During/After & $\mathbf{p} / \boldsymbol{\Delta}$ & Glycemia Pre/After & $\mathbf{p} / \boldsymbol{\Delta}$ \\
\hline $\mathrm{AF}$ & $169,9 / 118$ & $0,00 / 51,9$ & $118 / 117,3$ & $1,0 / 0,7$ & $169,9 / 117,3^{*}$ & $0,00 / 52,6$ \\
\hline $\mathrm{FA}$ & $166,6 / 134,7^{*}$ & $0,00 / 31,9$ & $134,7 / 118,4$ & $0,01 / 16,3$ & $166,6 / 118,4^{*}$ & $0,00 / 48,2$ \\
\hline
\end{tabular}

Note: Average value in mg/dl. AF: Aerobic Strength Training, AF: Aerobic Strength Training. Pre-Glycemia: Before to start training. Glycemia during half of the training. Blood glucose after at the end of the training. $P^{*}$ significant level. Variation of the delta.

\section{Discussion}

Based on the limitations of this study, it is suggested that new investigations be performed, using a larger sample and longitudinal follow-ups. According to the analyzed results, independent of the training order, there was a significant reduction in capillary glycemia of type 2 diabetics, when compared to the PRE/POST moment, although the PA order presented a greater reduction until the half of the session, this difference was not significant. In the study of Yardley et al. [12], who also studied the order of trainings, concluded that with higher blood glucose, one should start the session with aerobic training and when the blood glucose is lower one should start with strength training, because it tends to cause a less accentuated reduction of glucose, consequently, less risk of causing hypoglycemia, however, the study was conducted with individuals with type 1 diabetes. Savikj \& Zierath [18], in a review article, bring the treatment of DM2 as a complex issue in which the type of exercise, intensity and volume and nutritional status, offer great impact and should be considered.

When talking about the nutritional state, in the benefits associated to EF, the same study indicates that training with low carbohydrate availability can improve cardiorespiratory function and oxidative capacity of skeletal muscle compared to conventional training, but can also increase the chance of hypoglycemia, especially in DM2. The present study did not verify glycemic response with different volumes and intensities, and the participants maintained their routine feeding. Yang et al. [19] compared the effectiveness of aerobic training versus strength and verified, through a metaanalysis, that the aerobic showed greater reduction of glycated hemoglobin (HbA1C) after intervention, HbA1c is the average of three months of capillary blood glucose, an important marker for 
the control of dibetes1. However, as a limiting factor, most studies included were cross-sectional. Corroborating this same study, Schwingshackl et al. [20], conducted a meta-analysis showing greater effects of combined training in type 2 diabetics, but when compared aerobic training with strength training, aerobics showed better control in HbA1C. It is worth mentioning that this study evaluated capillary glycemia and not $\mathrm{HbA} 1 \mathrm{c}$ and it was not possible to observe greater benefits among the evaluated protocols.

According to Crisafulli et al. [21] individuals with DM types 1 and 2 experience different problems in hemodynamic regulation during exercise, as a possible deregulation in cardiovascular response, therefore, may lead to different responses in training, which reinforces the need for research involving individuals with type 2 diabetes, in view of previous responses in individuals with type 1 diabetes [13]

\section{Conclusion}

It is concluded that the combined training, in the order of AF or FA, was safe and efficient in the physical training of individuals with type 2 diabetes. However, the intragroup analysis did not show any difference in glycemic modulation in any of the orders, allowing the Physical Education professional greater freedom in choosing the order of exercises during the prescription of physical exercises for this public.

\section{References}

1. (2019) Brazilian Society of Diabetes: Guidelines of the Brazilian Diabetes Society. [S.L.]: ( $1^{\text {st }}$ Edn). São Paulo, Clannad pp. 30 - 490.

2. (2016) Guidelines of the Brazilian Diabetes Society. Organization José Egidio Paulo de Oliveira, Sérgio Vencio-São Paulo: AC Farmacêutica.

3. Hamasaki H (2016) Daily physical activity and type 2 diabetes: a review. ( $7^{\text {th }}$ edn). World J Diabetes 7(12): 243-251.

4. Vancea Dmm, Vancea Jn, Pires Mif, Reis Ma, Moura Rb, et al. (2009) Effect of frequency of physical exercise on glycemic control and body composition in type 2 diabetics. ( $1^{\text {st }}$ edn). Brazilian Archives of Cardiology (Print) 92(1): 23-30

5. Tang JL, Li XY, Yuan L, Han J, JU CP, et al. (2015) Correlation of regular physical exercise with metabolic control and reduction of chronic complications in type 2 diabetic patients. Chin J Diabetes Mellitus 136141.

6. thent ZC, Das S, Henry jj (2013) Role of exercise in the management of diabetes 3: 0 mellitus: the global scenario. ( $1^{\text {st }}$ edn). Plos One.
7. Colberg SR, Sigal RJ, Yardley JE, Michael C Riddell, David W Dunstan, et al. (2016) Physical Activity/Exercise and Diabetes: A Position Statement of the American Diabetes Association. Diabetes Care. (1 ${ }^{\text {st }}$ edn) 39(11): 2065-2079.

8. Cao L, LI X, YAN P, Xiaoqin Wang, Meixuan Li MM, et al. (2019) The effectiveness of aerobic exercise for the hypertensive population: a systematic review and meta-analysis. J Clin Hypertens 21: 868-876.

9. (2017) Wang Y, XU D. Effects of aerobic exercise on lipids and lipoproteins. Lipids Health Dis 16: 132.

10. (2019) American Diabetes Association. Standards of medical care in diabetes. ( $1^{\text {st }}$ edn), Diabetes Care.

11. Jenkins DW, Jenks A (2017) Exercise and Diabetes: A Narrative Review. (1 $1^{\text {st }}$ edn). J Foot Ankl Surg, 56(5): 968-974

12. Ribeiro JNS, Lima AMB, Franca JAL, Silva VNS, Cavalcanti CBS, Vancea DMM. (2017) Doce Vida - Supervised Physical Exercise Program for Diabetics ( $1^{\text {st }}$ edn) Rev Andaluza de Med Del Deporte 147:1.

13. Jane E. Yardley, Glen P. Kenny, Bruce A Perkins, Michael C. Riddell, Janine Malcolm, et al. (2012) Effects of Performing Resistance Exercise Before Versus After Aerobic Exercise on Glycemia in Type 1 Diabetes ( $1^{\text {st }}$ edn). Diabetes Care 35(4): 669-675.

14. (2017) Fleck SJ, Kraemer WJ. Fundamentals of muscle strength training. $\left(4^{\text {th }}\right.$ edn) Porto Alegre: Artmed.

15. Marins JCB, luiz A, monteiro A, jesus G (1998) Validation of the heart rate measurement time after the maximum effort of 50 and $80 \%$. Rev Bras Med Esporte 4(4): 114 - 119.

16. Perez AJ, Dias KD, Carletti L (2010) Intensity control of aerobic exercise by palpation of the radial art. Rev.Bras.Cineantropométrico Performance 12(3): 186-194.

17. Johanna Hortensius, Robbert J Slingerland, Nanne Kleefstra, Susan J] Logtenberg, Klaas H Groenier, et al. (2011) Self-Monitoring of blood glucose: The Use of the First or the Second Drop of Blood. ( $1^{\text {st }}$ edn). Diabetes Care 34(3): 556-560.

18. Savikj M, Zierath JR (2020) Train like an athlete: applying exercise interventions to manage type 2 diabetes ( $1^{\text {st }}$ edn) Diabetologia 14911499.

19. Yang Z, Scott CA, Mao C, Tang J, Farmer AJ (2010) Resistance exercise versus aerobic exercise for type 2 diabetes: a systematic review and meta-analysis. (1 ${ }^{\text {st }}$ edn) Sports Med 44(4): 487-499.

20. Schwingshackl L, Missbach B, Dias S, König J, Hoffmann G (2014) Impact of different training modalities on glycemic control and blood lipids in patients with type 2 diabetes: a systematic review and network metaanalysis. (1 $1^{\text {st }}$ edn) Diabetologia 57(9): 1789-1797.

21. Roberto S, Crisafulli A (2017) Consequences of Type 1 and 2 Diabetes Mellitus on the Cardiovascular Regulation During Exercise: A Brief Review (1 ${ }^{\text {st }}$ edn) Curr Diabetes Rev 13(6): 560-565. 